ARTICLE OPEN

\title{
Temporal dynamics of bacterial microbiota in the human oral cavity determined using an in situ model of dental biofilms
}

Nanako Wake ${ }^{1,7}$, Yoko Asahi ${ }^{1,7}$, Yuichiro Noiri ${ }^{2}$, Mikako Hayashi $^{1}$, Daisuke Motooka ${ }^{3}$, Shota Nakamura ${ }^{3}$, Kazuyoshi Gotoh ${ }^{4}$, Jiro Miura ${ }^{5}$, Hiroyuki Machi ${ }^{6}$, Tetsuya lida ${ }^{3}$ and Shigeyuki Ebisu ${ }^{1}$

Numerous studies on oral biofilms have been performed in vitro, although it is difficult to mimic the oral environment. Here we used an in situ model to conduct a quantitative analysis and comprehensive identification of bacterial communities over time by performing deep sequencing of 16S rRNA genes. We show here that the number of viable bacteria in supragingival biofilms increased in two steps. Using scanning and transmission electron microscopy, as well as confocal laser scanning microscopy, we detected gram-positive cocci during the first $8 \mathrm{~h}$. The biofilm was subsequently covered with a thick matrix-like structure composed of different bacterial morphotypes that diversified as the number of bacteria increased. Streptococcus accounted for $>20 \%$ of the population until $16 \mathrm{~h}$, and obligate anaerobes such as Fusobacterium, Prevotella and Porphyromonas predominated after $48 \mathrm{~h}$, and this increase was statistically significant after $96 \mathrm{~h}(P<0.05)$. Together, our data demonstrate that an initial population of facultative anaerobic bacteria was replaced with a population of gram-negative anaerobic bacteria during oral biofilm formation. This study, therefore, contributes to a comprehensive understanding of the composition of the bacterial microbiota involved in the health of the human oral cavity.

npj Biofilms and Microbiomes (2016) 2, 16018; doi:10.1038/npjbiofilms.2016.18; published online 10 August 2016

\section{INTRODUCTION}

Biofilms are complex structures cooperatively formed by bacterial communities comprising multiple species that grow on a solid surface. ${ }^{1}$ The oral cavity is inhabited by $\geqslant 700$ species of bacteria ${ }^{2,3}$ that form dental biofilms in various places such as the tooth surface and gingival sulcus. Biofilm formation is affected by diverse intraoral environmental and host factors such as host immunity, pH, enzymes, saliva and antibiotics. ${ }^{4}$ Biofilms are a major cause of caries and periodontal disease, and the interaction of biofilm microbial populations affects bacterial virulence. ${ }^{5}$ Therefore, it is important to understand the mechanism of biofilm formation to prevent and treat dental diseases. Previous studies of dental biofilms investigated the mechanism of biofilm formation, gene expression and exclusion-suppression methods using in vitro models that used one or a few bacterial species. ${ }^{6-10}$ However, dental biofilms are difficult to model in vitro because of their species diversity and the complex environment of the oral cavity. Therefore, in situ models are required to understand the mechanism of human dental biofilm formation and how to mitigate their contribution to disease.

Previous analyses utilized in situ models of biofilm formation. ${ }^{11,12}$ However, these studies were primarily qualitative, relying on microscopic observations of biofilm formation, and the data are insufficient to acquire a detailed understanding of the mechanism of dental biofilm formation. Further, previous studies found that dental biofilm formation increases bacterial diversity and alters the predominant bacterial species present over time, which changes the environment in which oral disease develops. ${ }^{6,13}$ To understand the causes of oral diseases, the dynamics of bacterial communities in biofilms must be investigated using quantitative and metagenomic analyses. In situ models of biofilms offer a unique opportunity to perform these investigations.

Recently, much effort has been invested in understanding the communities of bacteria that inhabit the interior and surface of the human body. The 2012 Human Microbiome Project conducted a large-scale metagenomic analysis of the microbiota of the airway, skin, oral cavity, gut stool and vagina of healthy adults. ${ }^{14}$ This study revealed the characteristics of the normal microbial populations of healthy individuals for the first time and clarified the role of variations in the normal microbiota in human health and disease.

The Human Microbiome Project investigated the composition of the bacterial microbiota on the tongue surface, buccal mucosa, saliva, supragingival plaque and subgingival plaque. ${ }^{15}$ Nextgeneration sequencing was used to compare the oral microbiota of people with or without caries, ${ }^{16}$ as well as the subgingival bacterial microbiota of periodontal tissue of normal subjects and with subjects with gingivitis or periodontitis; ${ }^{17}$ however, these analyses were conducted at a single time point. Therefore, insufficient data are available to understand the dynamics of bacterial microbiota over time. Similarly, studies that used in situ models and 16S rRNA gene sequencing comprehensively identified bacterial species that reside in biofilms; ${ }^{18,19}$ however,

\footnotetext{
${ }^{1}$ Department of Restorative Dentistry and Endodontology, Osaka University Graduate School of Dentistry, Osaka, Japan; ${ }^{2}$ Division of Cariology, Operative Dentistry and Endodontics, Department of Oral Health Science, Niigata University Graduate School of Medical and Dental Sciences, Niigata, Japan; ${ }^{3}$ Research Institute for Microbial Diseases,

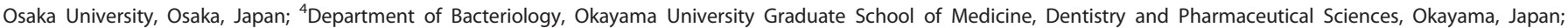
${ }^{5}$ Division for Interdisciplinary Dentistry, Osaka University Dental Hospital, Osaka, Japan and ${ }^{6}$ Osaka University Dental Technology Institute, Osaka, Japan.

Correspondence: Y Asahi (yoko-a@dent.osaka-u.ac.jp)

${ }^{7}$ These authors contributed equally to this work.

Received 8 February 2016; revised 7 June 2016; accepted 8 June 2016
} 
we are unaware of any detailed investigations that addresses the changes in bacterial species over time during biofilm maturation.

To address this gap in our knowledge, here we developed an in situ biofilm model and used it to analyse biofilms to evaluate the characteristics of human dental biofilms over time. We used our in situ model of biofilms to conduct a quantitative nextgeneration sequencing analysis of the microbiota of experimental biofilms and comprehensively identified changes in bacterial communities over time.

\section{RESULTS}

The population of biofilm-forming bacteria and the thickness of biofilms increase in two steps

Viable cell counts under aerobic conditions increased rapidly during the first $12 \mathrm{~h}$ and increased gradually thereafter. After a statistically significant increase between 48 and $72 \mathrm{~h}$, the population of viable cells plateaued (Figure 1a). Thus, the number of viable biofilm-forming cells increased in two steps. We used real-time PCR to simultaneously determine the number of bacteria present at each time (Figure $1 \mathrm{~b}$ ). First, the number of bacteria increased significantly from 1 to $12 \mathrm{~h}(P=0.0112)$ and increased gradually thereafter. The total number of bacteria increased again after $72 \mathrm{~h}$ and then plateaued. These results indicate that the increase in the total number of bacteria in a dental biofilm was similar to that of viable cells shown in Figure $1 \mathrm{a}$.

After $8 \mathrm{~h}$, biofilms were observed using confocal laser scanning microscope. Biofilm thickness increased after $24 \mathrm{~h}$. At $48 \mathrm{~h}$, the observed biofilm-forming area was expanded, and dead cells were frequently observed in the lower layers nearest the hydroxyapatite (HA) disk, and the number of live cells was increased in the upper layers (Figure 2a). Biofilm thickness increased and attained its maximum thickness $(50 \mu \mathrm{m})$ at this time. Biofilm thickness decreased slightly at $72 \mathrm{~h}$ (Figure $2 \mathrm{~b}$ ). Thus, the changes in biofilm thickness were similar to those of the viable cell count.

The volumes of live and dead cells changed similarly compared with the number of viable cells (Figure $2 \mathrm{~b}$ ). The volume of live cells increased rapidly during the first $24 \mathrm{~h}$ and then increased gradually until $72 \mathrm{~h}$ when the volumes of live and dead cells decreased slightly.

\section{Adherent biofilms formed on the disks}

The scanning electron microscope (SEM) and transmission electron microscope (TEM) observations performed after $8 \mathrm{~h}$ revealed the presence of biofilms comprising gram-positive cocci (Figures $3 \mathrm{a}$ and $\mathrm{b}$ ), and filamentous bacteria appeared after $12 \mathrm{~h}$ (Figure 3b). Subsequently, a thick matrix-like structure covered the biofilm (Figure 3a), which contained different morphotypes such as spherical, fusiform (Figure $3 b$ ) and filamentous (Figure $3 b$ ) bacteria. Moreover, dead cells called bacterial ghosts were frequently observed on the side of the HA disk (Figure 3b).

Oral bacterial diversity changes with time in healthy individuals We next determined the change in bacterial biofilm populations over time. Similarities in bacterial populations were detected among the 10 subjects (Supplementary Figure S1). For example, the phylum Firmicutes was dominant initially and Fusobacteria and Bacteroidetes increased after $48 \mathrm{~h}$ (Figure 4a). The phylum Proteobacteria was the most abundant except at $1 \mathrm{~h}$. Firmicutes accounted for $>30 \%$ of the bacterial population. After $48 \mathrm{~h}$, the relative abundance of Bacteroidetes and Fusobacteria increased, whereas that of Firmicutes and Actinobacteria decreased. Compared with their numbers at $1 \mathrm{~h}$, the increase of Bacteroidetes and Fusobacterium and the decrease of Firmicutes and Actinobacteria at $96 \mathrm{~h}$ were significantly different $(P<0.05)$.
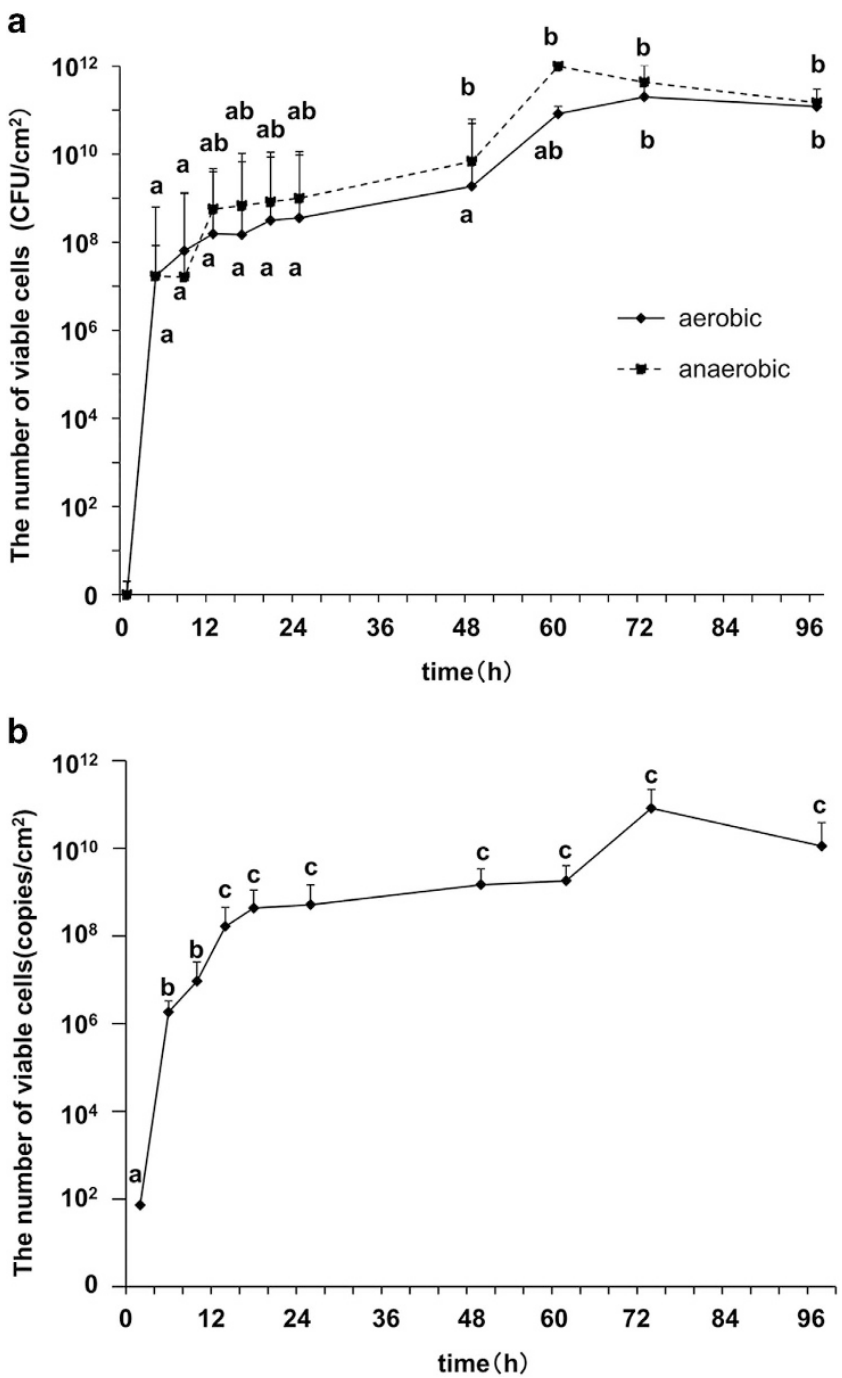

Figure 1. Quantification of biofilm-forming cells. (a) Viable bacterial cell count per unit area. Solid and dashed lines indicate bacteria incubated under aerobic or anaerobic conditions, respectively. The letters $\mathrm{a}$ and $\mathrm{b}$ represent a significant difference between the opposing letters $(P<0.05)$. Significant differences in the numbers of bacteria incubated under aerobic or anaerobic conditions are shown below the solid line and above dotted line, respectively. (b) Real-time PCR analysis of biofilm-forming cells. The letters a, b and $\mathrm{c}$ represent significant difference between the opposing letters (one-way analysis of variance, Tukey-Kramer test, $P<0.05$ ). Data are presented as the mean \pm s.e.m. $(n=10)$.

Firmicutes was represented almost entirely by Streptococcus; Fusobacteria was almost entirely composed of Fusobacterium and Bacteroidetes was represented by Capnocytophaga, Prevotella and Porphyromonas (Figure 4b). Therefore, our further analyses focused on temporal changes of the most frequently detected taxa, which averaged $>1 \%$ of the population (Supplementary Table S2). Together, aerobic and facultative anaerobic bacteria represented approximately $70 \%$ of recovered taxa until $24 \mathrm{~h}$. After $48 \mathrm{~h}$, the proportion of these taxa was reduced to $50-60 \%$. Conversely, anaerobic bacteria accounted for $\leqslant 20 \%$ of the taxa until $24 \mathrm{~h}$ and increased to $30-40 \%$ after $48 \mathrm{~h}$.

To determine whether these changes were statistically significant, we restricted our analysis to the five most abundant genera that represented $\geqslant 5 \%$ of the taxa (Figure $4 c$ ). In the initial phase of biofilm formation, Streptococcus accounted for $\geqslant 20 \%$ of the taxa. After $48 \mathrm{~h}$, the relative abundance of obligate anaerobes 
a
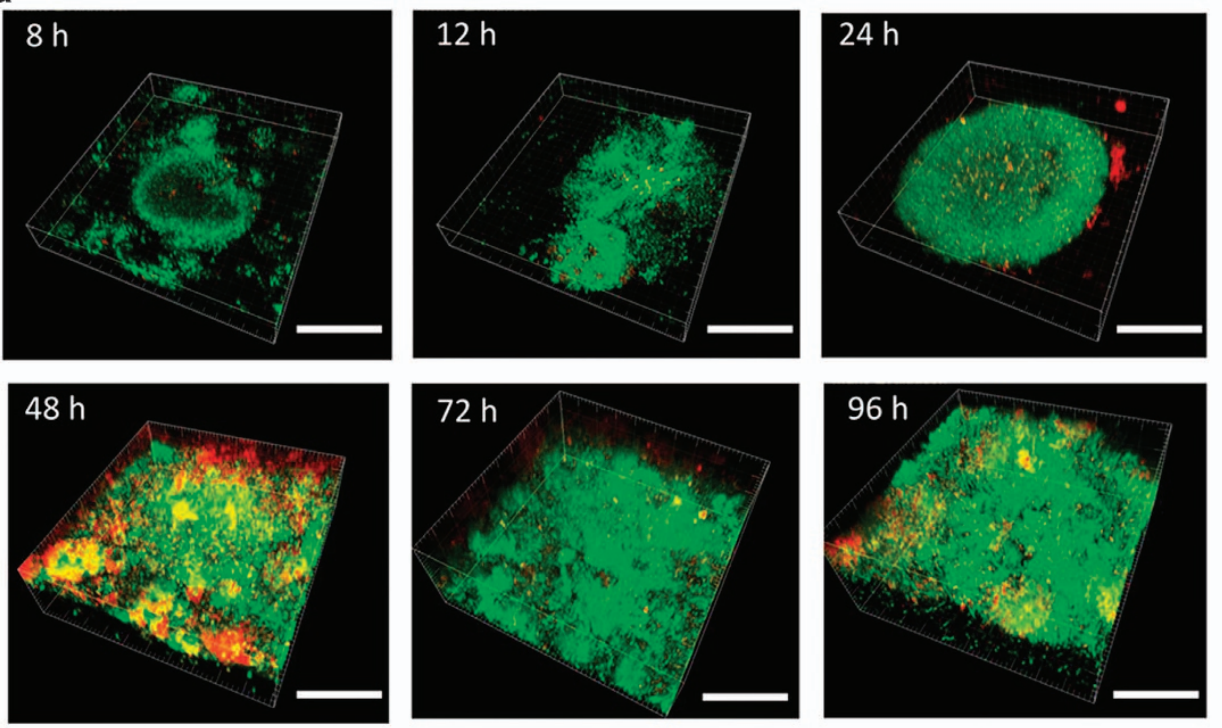

b

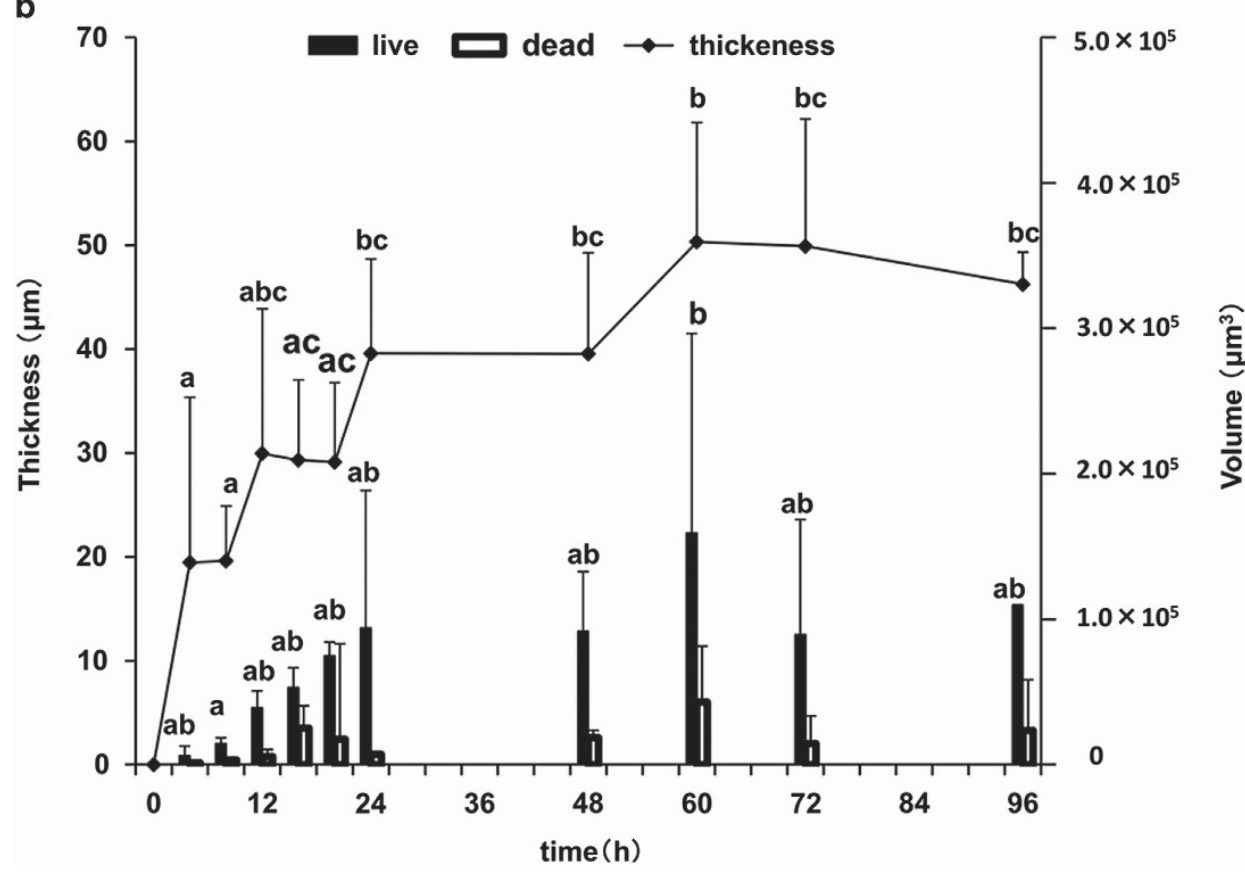

Figure 2. CLSM analysis of biofilms. (a) CLSM observations. Scale bar, $50 \mu \mathrm{m}$. (b) Determination of the thickness and volume of live and dead cells using Imaris imaging software. Black and white bars represent the volumes of live and dead cells, respectively. The letters a, $b$ and $c$ represent significant difference as indicated by the opposing letters (one-way analysis of variance, Tukey-Kramer test, $P<0.05$ ). There were no significant differences at any time between the volumes of dead cells. CLSM, confocal laser scanning microscope.

such as Fusobacterium, Porphyromonas and Prevotella increased significantly, and the difference was statistically significant after $96 \mathrm{~h}(P<0.05$; Figure $4 \mathrm{c})$. The population of Streptococcus was $\geqslant 20 \%$ until $16 \mathrm{~h}$, decreased starting at $24 \mathrm{~h}$ and was $<5 \%$ after $96 \mathrm{~h}$. In contrast, Neisseria accounted for $>20 \%$ after $4 \mathrm{~h}$, remaining constant thereafter. Fusobacterium began increasing in abundance after $48 \mathrm{~h}$, and was significantly increased at $96 \mathrm{~h}$ compared with $4 \mathrm{~h}(P=0.0380), 8 \mathrm{~h}(P=0.0233), 12 \mathrm{~h}(P=0.0207)$, $16 \mathrm{~h}(P=0.0122)$ and $24 \mathrm{~h} \quad(P=0.0340)$. The proportion of Porphyromonas began to increase after $48 \mathrm{~h}$ and was significantly higher at $96 \mathrm{~h}$ compared with $1 \mathrm{~h}(P=0.0265)$ and $16 \mathrm{~h}$ $(P=0.0480)$. Moreover, the proportion of Prevotella began to increase after $48 \mathrm{~h}$ and was significantly higher at $96 \mathrm{~h}$ compared with $4 \mathrm{~h}(P=0.0482), 8 \mathrm{~h}(P=0.0070), 12 \mathrm{~h}(P=0.0092)$ and $16 \mathrm{~h}$ $(P=0.0122)$.

\section{DISCUSSION}

The recent rapid development of techniques to analyse microbiomes has made possible major advances in the characterization of many human microbiomes, including the oral microbiota. ${ }^{14,15}$ However, in situ analyses of the population of biofilm-producing bacteria in the oral cavity only focus on the initial ${ }^{18}$ or later phase. ${ }^{19}$ Therefore, we used an in situ model of biofilm formation to investigate the temporal dynamics of the oral microbiome of healthy individuals. To our knowledge, the present study reveals 
a
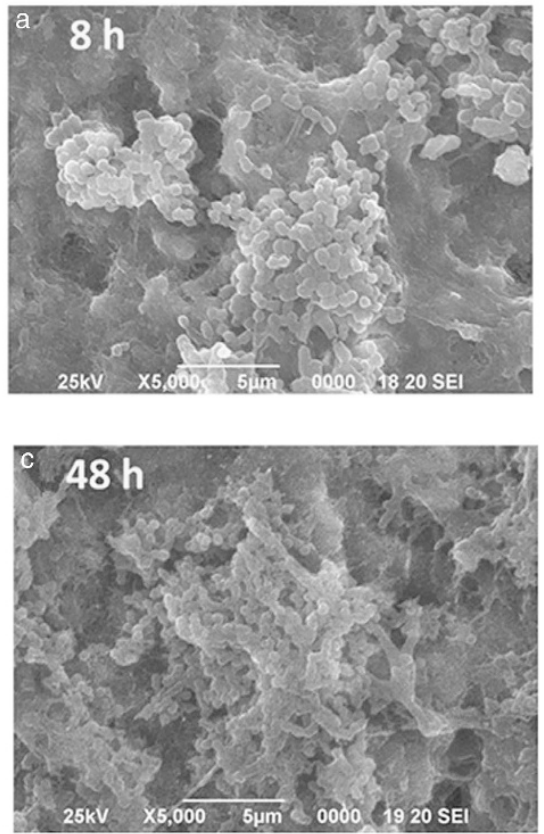

b
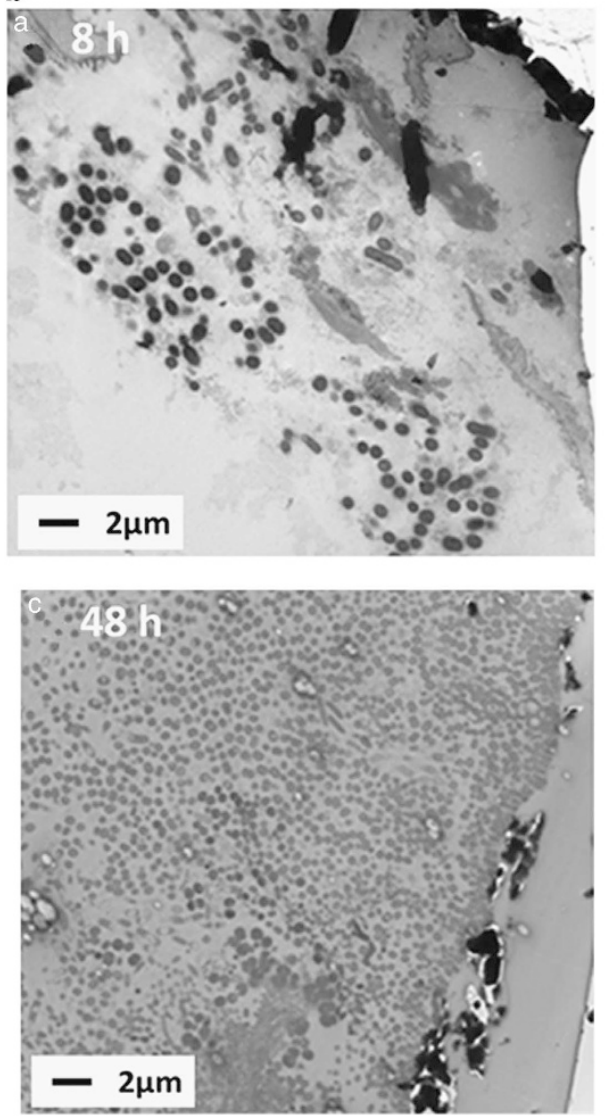
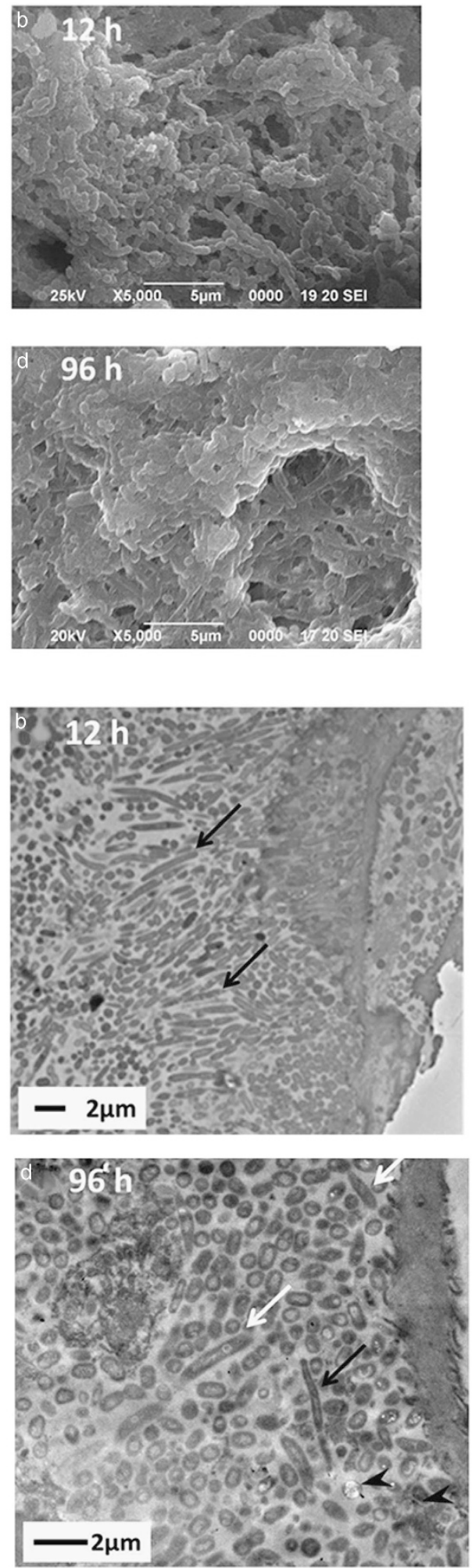

Figure 3. SEM and TEM observation of biofilms (a) SEM. (b) TEM. The side of the hydroxyapatite (HA) disk is shown on the right side of each image. Black arrow, filamentous bacteria; white arrow, fusiform bacteria; arrowhead, bacterial ghosts. SEM, scanning electron microscope; TEM, transmission electron microscope.

for the first time that the number of viable bacteria in a supragingival biofilm increased in two steps. For example, the populations of certain gram-negative anaerobic bacteria rapidly increased and predominated after $48 \mathrm{~h}$, which is consistent with sequencing data. Moreover, quantitative PCR analyses confirmed the biphasic increase in bacterial numbers (Figure 1). These findings are consistent with a previous quantitative analysis of biofilm formation on HA disks, which found that the 


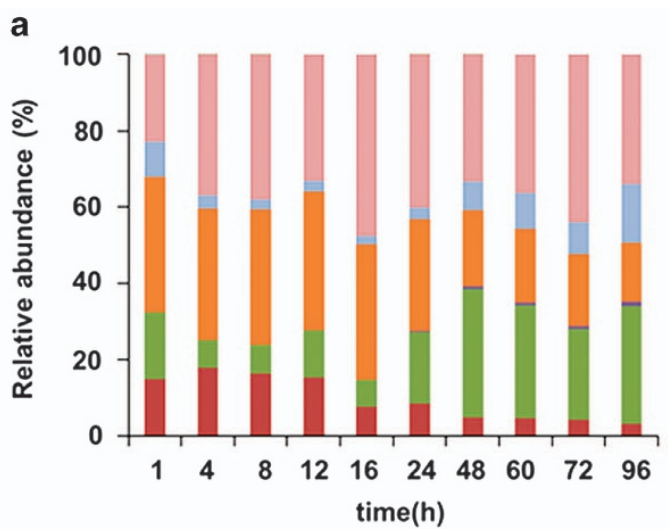

\begin{tabular}{l} 
Proteobacteria \\
$\begin{array}{ll}\text { Fusobacteria } & \text { Bacteroidetes } \\
\text { Firmicutes } & \text { Actinobacteria }\end{array}$ \\
\hline
\end{tabular}

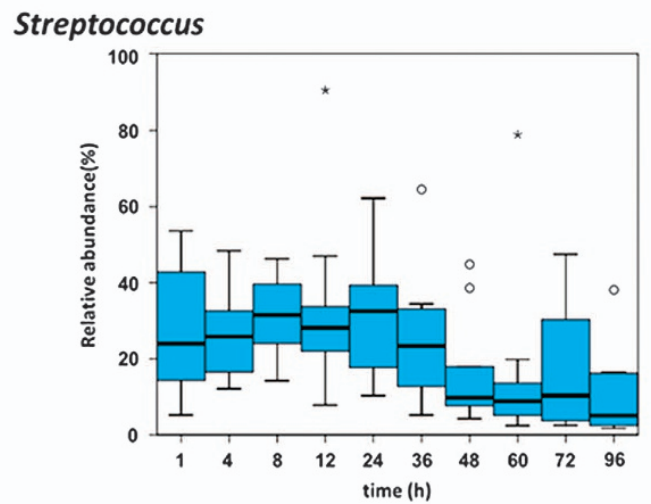

b

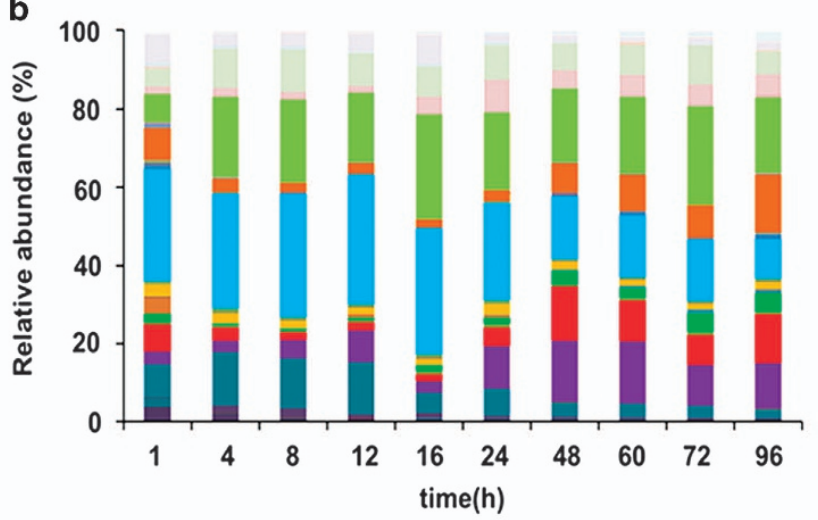

\begin{tabular}{|c|c|c|}
\hline Derxia & Fusobacterium & Bergeyella \\
\hline Sphingobacterium & - Veillonella & - Prevotella \\
\hline Haemophilus & = Streptococcus & - Porphyromonas \\
\hline Pasteurellaceae-other & Gemella & - Rothia \\
\hline Neisseria & - Flavobacterium & - Actinomyces \\
\hline Leptotrichia & - Capnocytophago & \\
\hline
\end{tabular}

Neisseria

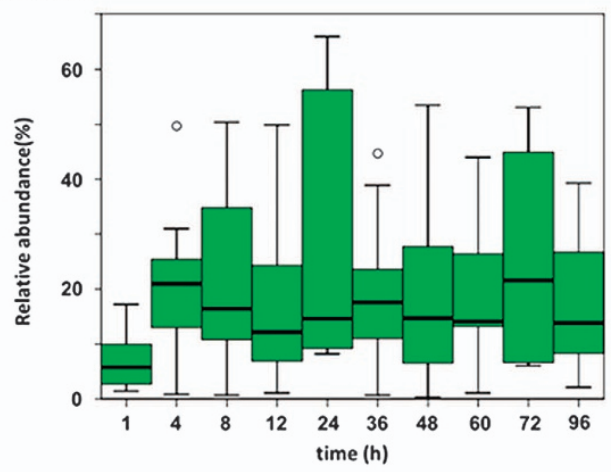

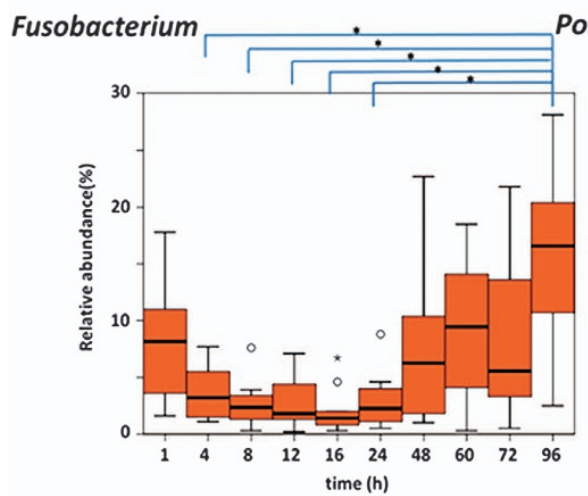

Porphyromonas
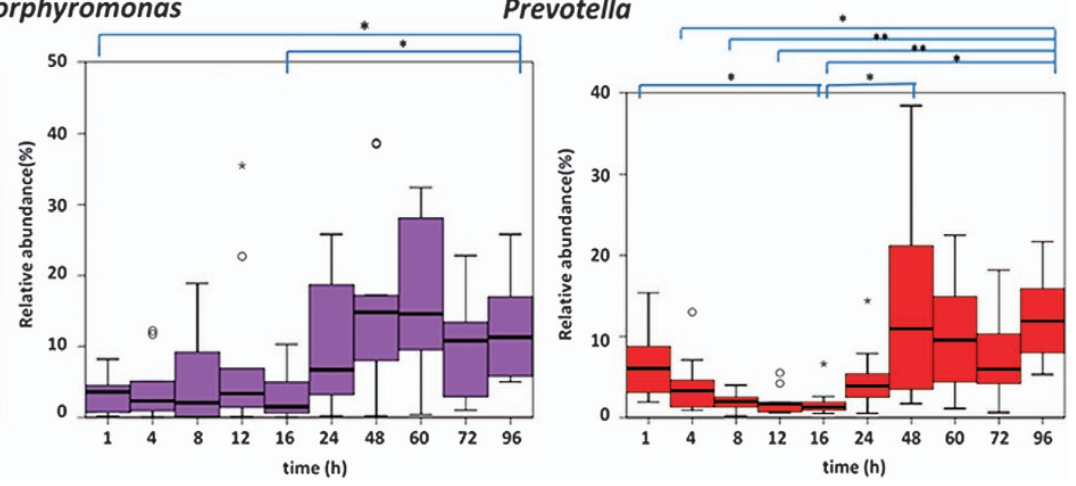

Figure 4. Relative abundances of bacterial taxa among the subjects. (a) Relative abundance of bacterial phyla in all subjects $(n=10)$. This graph represents the average of data collected from all the subjects at each time point. (b) Proportional abundance of genera in all the subjects $(n=10)$. The temporal changes in the relative amounts of each genus are indicated. The most frequently detected taxa $(>1 \%$ relative abundance) in each level are shown. (c) Proportional abundance of five genera. Boxes extend from the 25 th to 75 th percentiles. Circles represent outliers. Asterisks above the whisker indicate a statistically significant difference at each time. ${ }^{*} P<0.05 ;{ }^{* *} P<0.01$ (Kruskal-Wallis, Steel-Dwass test).

number of bacteria deposited on a biofilm reaches a plateau after 4 days. ${ }^{19}$

Further, the thickness and volume of biofilms revealed that these temporal changes correlated with temporal changes in the number of viable bacteria (Figure 2b). However, during one period of biofilm formation, thickness did not increase with an increase in viable cell counts, which we determined was caused by the increase in the surface area of biofilms. These results suggest that dental biofilms first increase in thickness and then in area, which is followed by a further increase in thickness. After $60 \mathrm{~h}$, the thickness and volume of live and dead bacteria decreased, although the biofilm covered the entire area of the HA disks. It is therefore likely that dental biofilms peeled off during this period. This may be explained by reaching a thickness limit determined 
a

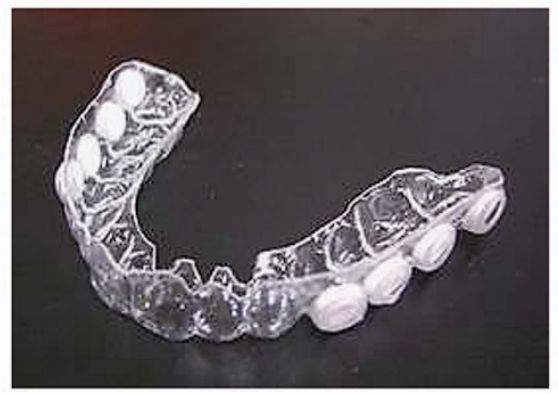

b

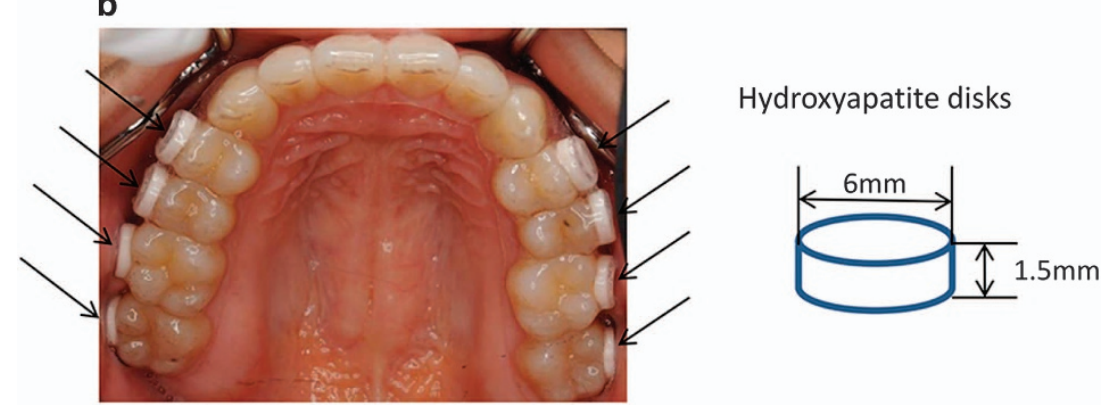

Figure 5. Image of upper-jaw acrylic appliance with eight hydroxyapatite (HA) disks. The diameter of the disks was 6 mm. The disks were fixed in the region of the premolars and molars toward the natural teeth on the buccal sides (arrows). (a) Appliance. (b) Appliance worn by a subject.

by a biofilm's three-dimensional structure. For example, the dead and viable bacterial cells were observed in the lower and upper layers, respectively, after $48 \mathrm{~h}$. This finding is consistent with previous studies ${ }^{20}$ and indicates the importance of dead cells and debris in the initial stages of biofilm development.

Here, we sampled during three consecutive time intervals. One report that used nucleotide sequence analysis shows that in initial dental biofilms, there is no significant difference in the percentage of bacteria constituting the biofilm sampled at different day. ${ }^{18}$ In contrast, we are not aware of any studies that focus on mature biofilms. In the present study, when we collected samples at $12 \mathrm{~h}$ during the primary and secondary periods and at $48 \mathrm{~h}$ during the secondary and third periods, we found that there was no significant difference between the bacterial compositions of each (data are not shown). Therefore, we concluded that bias was not introduced when samples were acquired at different intervals within each of the three periods.

To determine whether our in situ approach accurately modelled biofilm formation in vivo, we performed SEM and TEM analyses (Figure 3). The artificial biofilms were morphologically similar to those that adhere to natural tooth surfaces. ${ }^{21,22}$ Further, the bacterial morphologies observed using TEM, which correlated with our sequencing data, demonstrate that until $12 \mathrm{~h}$, the biofilms were primarily populated by gram-positive cocci, and after $48 \mathrm{~h}$, we observed diverse bacterial morphologies.

Previous studies demonstrate that maturation of dental plaques correlates with increased bacterial diversity over time. ${ }^{23}$ However, we show here that bacterial diversity decreased during the first $16 \mathrm{~h}$, subsequently increased, and became most diverse after $96 \mathrm{~h}$ (Supplementary Figure S2). It is considered that because the partial pressure of oxygen in biofilms decreases as they mature, the proportion of aerobic bacteria is reduced until $16 \mathrm{~h}$, and only aerotolerant bacteria such as Streptococcus remained. Diversity increased thereafter when the biofilm became anaerobic, and anaerobic bacteria were more likely to adhere. It is possible that the accumulation of metabolites produced by aerotolerant bacteria such as Streptococcus, or an insufficient nutrient supply, decreased bacterial diversity during the initial phase of biofilm formation. Further investigations of environmental factors such as oxygen pressure, nutrients and $\mathrm{pH}$ are required to understand the changes in the bacterial diversity of biofilms.

Our sequence analysis of bacterial taxa identified variations between healthy subjects, which is consistent with previous reports. $^{24,25}$ However, when we performed statistical analysis, certain trends were observed (Figure 4c). For example, the prevalence of Streptococcus during the initial phase of biofilm formation was consistent with previous investigations of initial communities, which used an in situ model. ${ }^{18,24}$ The phenotypes of streptococcal species, which enable them to become the pioneer species during biofilm formation, include their efficient attachment to the pellicle of enamel, ${ }^{26,27}$ as well as their ability to directly metabolize components of saliva as a nutrient source. ${ }^{28}$ Thus, it is likely that Streptococcus preferentially adhered to disks fabricated from hydroxyapatite, which is the main component of enamel.

Further Diaz et al. ${ }^{18}$ demonstrated that during the first $8 \mathrm{~h}$ of biofilm formation, Streptococcus, Gemella and Neisseria were commonly detected as the group of initial bacteria in three subjects. We show here that the genera Streptococcus and Neisseria were dominant in the early phase and that $48 \mathrm{~h}$ after the initiation of biofilm formation, gram-negative anaerobic bacteria such as Fusobacterium, Prevotella and Porphyromonas predominated (Figure 4). These findings are consistent with three studies of mature plaque. ${ }^{6,19,29}$ For example, Ritz et al. ${ }^{29}$ investigated the temporal changes in the microbial composition of supragingival biofilms cultured for 9 days and found that the aerobic and facultative anaerobic bacteria Streptococcus and Neisseria predominated, whereas the proportion of Fusobacterium increased after 9 days. Moreover, recent molecular investigations of oral microbiota demonstrate variations in bacterial microbiota during the maturation of dental biofilms, ${ }^{5,30,31}$ consistent with our present results. Moreover, the shift in bacterial microbiota from aerobic to anaerobic conditions during biofilm formation may be attributed to the creation of niches that are beneficial for the proliferation and survival of obligate anaerobic bacteria. ${ }^{18}$ 


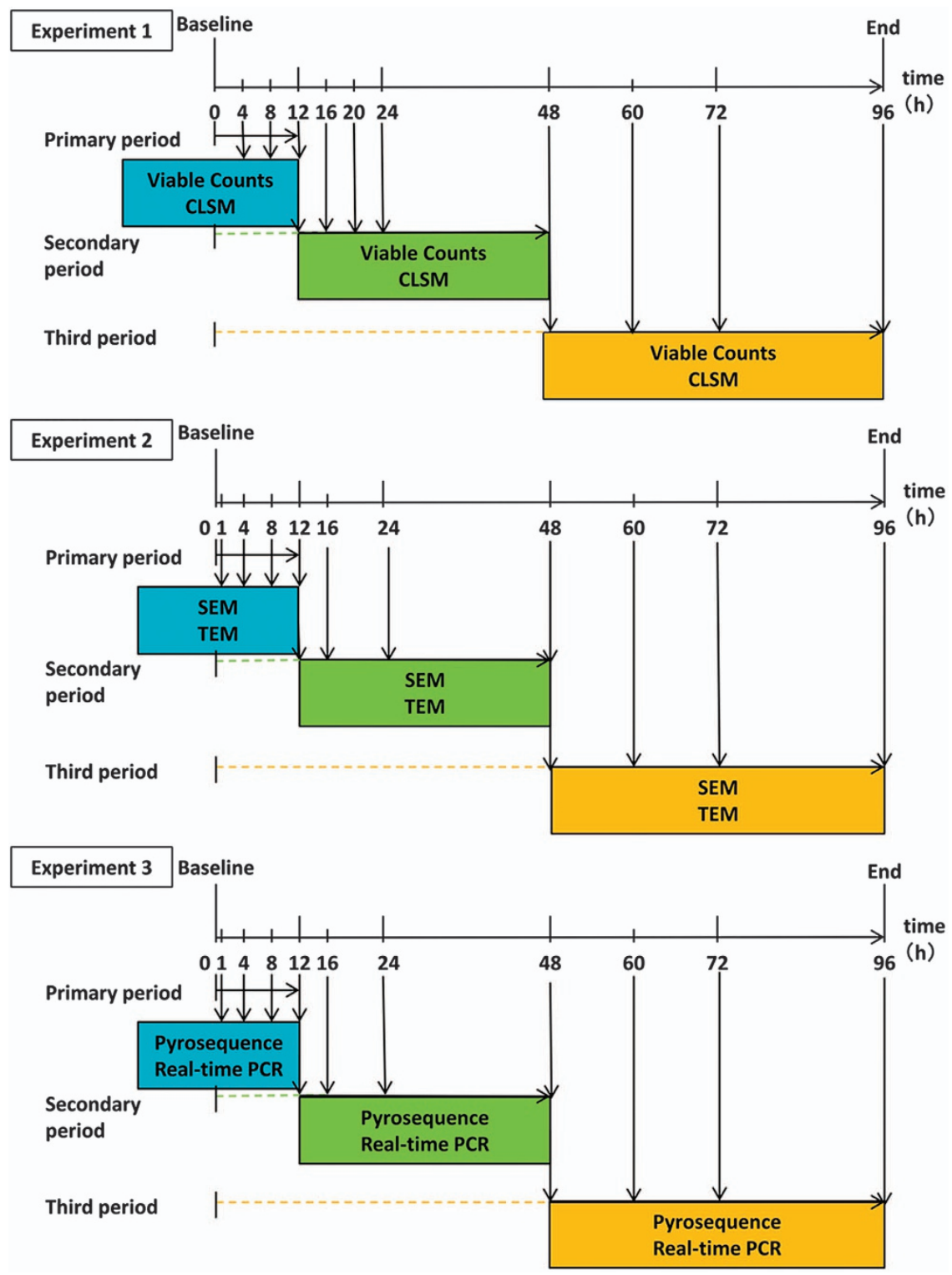

Figure 6. Experimental protocol. Experiment 1: determination of viable counts and three-dimensional analysis. Experiment 2: SEM and TEM observations. Experiment 3: DNA sequencing and real-time PCR quantification of biofilm-forming cells. CLSM, confocal laser scanning microscope; SEM, scanning electron microscope; TEM, transmission electron microscope; Viable counts, determination of viable count.

A study that investigated the effect of the time interval between brushing one's teeth and the induction of gum inflammation found that brushing every $48 \mathrm{~h}$ increases plaque scores, although gingivitis does not develop. ${ }^{32}$ The authors speculated that this may be explained by the quantitative and qualitative changes in biofilms that occur approximately $48 \mathrm{~h}$ after initial growth. Our results agree with this finding. Thus, we show here that the proportion of anaerobic bacteria that cause gingivitis, such as Fusobacterium, Prevotella and Porphyromonas, ${ }^{31,33}$ increased after $48 \mathrm{~h}$, indicating a shift to an environment that aggravates gingivitis. Thus, owing to the temporal variations of oral microbiota, we recommend that teeth should be brushed at least once every $48 \mathrm{~h}$ to prevent exacerbating gingivitis.

In conclusion, we used an in situ model of biofilm formation and detected a biphasic change in the phyla of biofilm-forming bacteria in the oral cavity of healthy subjects. Further, the bacterial population converted from facultative anaerobes to gramnegative anaerobes. It will be useful to compare the results of the present study with time-dependent changes in samples collected from individuals suffering caries or periodontal disease. Such studies will likely contribute to a comprehensive understanding of the bacterial microbiota involved in the health of the human oral cavity.

\section{MATERIALS AND METHODS}

Study subjects

The study included 10 healthy volunteers (three men and seven women) aged 26-30 years (mean 27.1 \pm 1.2 years) who were students of the Osaka University Graduate School of Dentistry during the study. We defined healthy volunteers as previously reported. ${ }^{34}$ Clinical or radiological signs of caries, gingivitis or periodontitis were not detected in any of the subjects. The volunteers abstained from antibiotics 6 months before the study commenced, and volunteers signed an informed consent form. The Ethics Committee of the Osaka University Graduate School of Dentistry approved the study design (H24-E4).

\section{In situ model of biofilms}

All volunteers wore a custom acrylic splint in their upper jaw for $96 \mathrm{~h}$ to form mature dental biofilms. In the splint, eight HA disks $(6 \mathrm{~mm}$ diameter, 
$1.5 \mathrm{~mm}$ height) were inserted on the buccal side so that the biofilms were not peeled off by the tongue or cheek (Figure 5). The volunteers wore this splint for $96 \mathrm{~h}$, except during meals and while brushing their teeth as previously described. ${ }^{35}$ The appliance was stored in a humidified chamber. After dental biofilms were formed, the disks were extracted without disrupting the adherent biofilm.

\section{Experimental protocol}

Dental biofilms formed on the HA disks were evaluated at 1, 4, 8, 12, 16, 24, $48,60,72$ and $96 \mathrm{~h}$. Because only eight disks fit into the appliance, we pooled our samples into three periods as follows: primary period (0-12 h), secondary period (12-48 h) and third period (48-96 h; Figure 6). At 1, 4, 8, $12,16,24,48,60,72$ and $96 \mathrm{~h}$, two disks were extracted and used for two assays, for example, one disk was used to determine the number of viable bacteria and the other was used for confocal laser scanning microscope in Experiment 1.

\section{Determination of viable count}

The samples were immersed in sterile distilled water, sonicated for 5 min and vortexed for $30 \mathrm{~s}$. The specimens were used to inoculate Colombia blood agar (Becton, Dickinson and Company, Fukushima, Japan) and incubated aerobically or anaerobically for $48 \mathrm{~h}$. Determination of viable counts was conducted by counting colonies. Each sample was tested in triplicate.

\section{Microscopy}

Biofilm samples stained with the LIVE/DEAD BacLight Bacterial Viability Kit (Invitrogen, Carlsbad, CA, USA) were observed using a confocal laser scanning microscope (LSM700; Carl Zeiss, Oberkochem, Germany), as previously described. ${ }^{36}$ After reconstruction of confocal laser scanning microscope images using Imaris imaging software (Imaris 5.0.1; Bitplane AG, Zurich, Switzerland), determination of the thickness and volumes of live cells and dead cells was performed.

\section{SEM observations}

The disks were prepared according to a previous protocol. ${ }^{36}$ The specimens were immersed in 50\% Karnovsky's solution $(2.5 \%$ glutaraldehyde, $2 \%$ paraformaldehyde) for $30 \mathrm{~min}$. They were then sequentially dehydrated in ethanol concentrations of 50,70, 80, 90, 95 and $100 \%$ and freeze-dried. After sputter-coating with platinum, the samples were observed using an scanning electron microscope (JSM-6390LV; JEOL, Tokyo, Japan).

\section{TEM observations}

Ultrastructural observations were performed as previously described. ${ }^{37}$ Briefly, the biofilms were fixed with $2.5 \%$ glutaraldehyde and $2 \%$ osmic acid, and dehydrated in a graded series of aqueous ethanol solutions followed by embedding in epoxy resin (Epon 812; NissinEM, Tokyo, Japan). Ultrathin sections were prepared and stained with $2 \%$ uranyl acetate and $0.4 \%$ Sato's lead stain. Images were acquired using a transmission electron microscope (H-800; Hitachi, Tokyo, Japan).

\section{DNA sequencing}

Two disks were collected at each time, and DNA was extracted using a PowerSoil DNA Isolation Kit (MO Bio Laboratories, Carlsbad, CA, USA). The V5-V6 region of the 16S rRNA gene was amplified using the primer set 784F: 5'-AGGATTAGATACCCTGGTA-3' and 1061R: 5'-CRRCACGAGCTGAC GAC- $3^{\prime} .^{38}$ Each library was prepared using an lon Fragment Library Kit (Life Technologies, Grand Island, NY, USA) according to the manufacturer's instructions. Sequencing was performed using an lon PGM Sequencing 400 Kit (Life Technologies) with the Ion PGM sequencer (Life Technologies). The resulting sequences were analysed using the QIIME pipeline. The processed sequences were then clustered into operational taxonomic units defined according to a similarity cutoff $=97 \%$ (see Supplementary Table S1) using UCLUST version 1.2.22q. Representative sequences for each operational taxonomic unit were classified taxonomically using RDP Classifier version 2.2 with the Greengenes Database. ${ }^{39}$

\section{Quantification of biofilm-forming cells}

We used real-time PCR to determine the number of the biofilm-forming cells according to a published method. ${ }^{40}$ Briefly, the assays were performed in a total volume of $20 \mu \mathrm{l}$ containing $10 \mu \mathrm{l}$ of SYBR Select Master Mix (Applied Biosystems, Carlsbad, CA, USA), $0.5 \mu$ l each of the forward and reverse 16S V5-V6 primer set universal primers (final concentration, $900 \mathrm{nM}$ each) and $1 \mu \mathrm{l}$ of DNA. The Applied Biosystems 7500 Fast real-time PCR system (Life Technologies) was used, and standard curves were analysed using the universal primer set to amplify a serial dilution of Streptococcus mutans NCTC10449 plasmid DNA. The experiments were repeated thrice for each sample. The data were analysed using the 7500 System SDS Software Version 2.0.2 (Applied Biosystems).

\section{Statistical analysis}

Differences in the number of viable cells, the thickness and the volumes of live and dead cells at each time point were evaluated using one-way analysis of variance and the Tukey-Kramer test implemented in JMP software (version10.0.2 2012 SAS Institute Inc., Cary, NC, USA). To perform statistical analysis of the temporal change of each genus, we used IBM SPSS Statistics (version 22.0, IBM SPSS Inc., Chicago, IL, USA) and the data are displayed as box plots. The differences between the populations of each genus at each time point were analysed using the Kruskal-Wallis and Steel-Dwass tests implemented in JMP software. Differences in the numbers of the biofilm-forming cells were analysed using one-way analysis of variance, Tukey-Kramer test. $P<0.05$ was considered significant.

\section{ACKNOWLEDGEMENTS}

We thank Masae Kuboniwa for helpful advice with the real-time PCR. This study was supported by Grants-in-Aid for Scientific Research (15H05021, 24390425, 26462878) by the Japan Society for the Promotion of Science.

\section{COMPETING INTERESTS}

The authors declare no conflict of interest.

\section{REFERENCES}

1. Costerton, J. W., Lewandowski, Z., Caldwell, D. E., Korber, D. R. \& Lappin-Scott, H. M. Microbial biofilms. Annu. Rev. Microbiol. 49, 711-745 (1995).

2. Kroes, I., Lepp, P. W. \& Relman, D. A. Bacterial diversity within the human subgingival crevice. Proc. Natl Acad. Sci. USA 96, 14547-14552 (1999).

3. Aas, J. A., Paster, B. J., Stokes, L. N., Olsen, I. \& Dewhirst, F. E. Defining the normal bacterial flora of the oral cavity. J. Clin. Microbiol. 43, 5721-5732 (2005).

4. Stewart, P. S. \& Costerton, J. W. Antibiotic resistance of bacteria in biofilms. Lancet 358, 135-138 (2001).

5. Socransky, S. S. \& Haffajee, A. D. Periodontal microbial ecology. Periodontol. 2000 38, 135-187 (2005).

6. Kolenbrander, P. E. et al. Bacterial interactions and successions during plaque development. Periodontol. 2000 42, 47-79 (2006).

7. Marsh, P. D., Moter, A. \& Devine, D. A. Dental plaque biofilms: communities, conflict and control. Periodontol. 2000 55, 16-35 (2011).

8. Yamaguchi, M. et al. Porphyromonas gingivalis mutant defective in a putative glycosyltransferase exhibits defective biosynthesis of the polysaccharide portions of lipopolysaccharide, decreased gingipain activities, strong autoaggregation, and increased biofilm formation. Infect. Immun. 78, 3801-3812 (2010).

9. Maezono, H. et al. Antibiofilm effects of azithromycin and erythromycin on Porphyromonas gingivalis. Antimicrob. Agents Chemother. 55, 5887-5892 (2011).

10. Yamamoto, R. et al. Time course of gene expression during Porphyromonas gingivalis strain ATCC 33277 biofilm formation. Appl. Environ. Microbiol. 77, 6733-6736 (2011).

11. Al-Ahmad, A. et al. The in vivo dynamics of Streptococcus spp., Actinomyces naeslundii, Fusobacterium nucleatum and Veillonella spp. in dental plaque biofilm as analysed by five-colour multiplex fluorescence in situ hybridization. J. Med. Microbiol. 56, 681-687 (2007).

12. Hannig, C., Follo, M., Hellwig, E. \& Al-Ahmad, A. Visualization of adherent micro-organisms using different techniques. J. Med. Microbiol. 59, 1-7 (2010).

13. Marsh, P. D. Dental plaque as a biofilm and a microbial community-implications for health and disease. BMC Oral Health 15, 6 (2006).

14. Human Microbiome Project Consortium. A framework for human microbiome research. Nature 486, 215-221 (2012).

15. Human Microbiome Project Consortium. Structure, function and diversity of the healthy human microbiome. Nature 486, 207-214 (2012). 
16. Yang, F. et al. Saliva microbiomes distinguish caries-active from healthy human populations. ISME J. 6, 1-10 (2012).

17. Park, O. J. et al. Pyrosequencing analysis of subgingival microbiota in distinct periodontal conditions. J. Dent. Res. 94, 921-927 (2015).

18. Diaz, P. I. et al. Molecular characterization of subject-specific oral microflora during initial colonization of enamel. Appl. Environ. Microbiol. 72, 2837-2848 (2006).

19. Takeshita, T. et al. Dental plaque development on a hydroxyapatite disk in young adults observed by using a barcoded pyrosequencing approach. Sci. Rep. 5, 8136 (2015).

20. García-Caballero, L. et al. Chlorhexidine substantivity on salivary flora and plaque-like biofilm: an in situ model. PLOS ONE 8, e83522 (2013).

21. Löe, H., Theilade, E. \& Jensen, S. B. Experimental gingivitis in man. J. Periodontol. 36, 177-187 (1965).

22. Theilade, E., Wright, W. H., Jensen, S. B. \& Löe, H. Experimental gingivitis in man. II. A longitudinal clinical and bacteriological investigation. J. Periodontal Res. 1, 1-13 (1966).

23. Rickard, A. H., Gilbert, P., High, N. J., Kolenbrander, P. E. \& Handley, P. S. Bacterial coaggregation: an integral process in the development of multi-species biofilms. Trends Microbiol. 11, 94-100 (2003).

24. Tinanoff, N., Gross, A. \& Brady, J. M. Development of plaque on enamel: parallel investigations. J. Periodontal Res 11, 197-209 (1976).

25. Li, J. et al. Identification of early microbial colonizers in human dental biofilm J. Appl. Microbiol. 97, 1311-1318 (2004).

26. Scannapieco, F. A. Saliva-bacterium interactions in oral microbial ecology. Crit. Rev. Oral Biol. Med. 5, 203-248 (1994).

27. Takahashi, Y., Konishi, K., Cisar, J. O. \& Yoshikawa, M. Identification and characterization of hsa, the gene encoding the sialic acid-binding adhesin of Streptococcus gordonii DL1. Infect. Immun. 70, 1209-1218 (2002).

28. Palmer, R. J. Jr, Kazmerzak, K., Hansen, M. C. \& Kolenbrander, P. E. Mutualism versus independence: strategies of mixed-species oral biofilms in vitro using saliva as the sole nutrient source. Infect. Immun. 69, 5794-5804 (2001).

29. Ritz, H. L. Microbial population shifts in developing human dental plaque. Arch. Oral. Biol. 12, 1561-1568 (1967)

30. Socransky, S. S. et al. Checkerboard DNA-DNA hybridization. Biotechniques 17, 788-792 (1994).
31. Socransky, S. S., Haffajee, A. D., Cugini, M. A., Smith, C. \& Kent, R. L. Jr. Microbial complexes in subgingival plaque. J. Clin. Periodontol. 25, 134-144 (1998).

32. Lang, N. P., Cumming, B. R. \& Löe, H. Toothbrushing frequency as it relates to plaque development and gingival health. J. Periodontol. 44, 396-405 (1973).

33. Moore, W. E. \& Moore, L. V. The bacteria of periodontal diseases. Periodontol. 2000 5, 66-77 (1994).

34. Aagaard, K. et al. The Human Microbiome Project strategy for comprehensive sampling of the human microbiome and why it matters. FASEB J. 27, 1012-1022 (2013).

35. Beyth, N., Yudovin-Farber, I., Perez-Davidi, M., Domb, A. J. \& Weiss, E. I. Polyethyleneimine nanoparticles incorporated into resin composite cause cell death and trigger biofilm stress in vivo. Proc. Natl Acad. Sci. USA 107, 22038-22043 (2010).

36. Asahi, Y. et al. Synergistic effects of antibiotics and an $\mathrm{N}$-acyl homoserine lactone analog on Porphyromonas gingivalis biofilms. J. Appl. Microbiol. 112, 404-411 (2012).

37. Asahi, Y. et al. Effects of the tea catechin epigallocatechin gallate on Porphyromonas gingivalis biofilms. J. Appl. Microbiol. 116, 1164-1171 (2014).

38. Andersson, A. F. et al. Comparative analysis of human gut microbiota by barcoded pyrosequencing. PLOS ONE 3, e2836 (2008).

39. DeSantis, T. Z. et al. Greengenes, a chimera-checked 16S rRNA gene database and workbench compatible with ARB. Appl. Environ. Microbiol. 72, 5069-5072 (2006).

40. Kuremoto, K. et al. Promotion of endodontic lesions in rats by a novel extraradicular biofilm model using obturation materials. Appl. Environ. Microbiol. 80, 3804-3810 (2014)

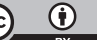

This work is licensed under a Creative Commons Attribution 4.0 International License. The images or other third party material in this article are included in the article's Creative Commons license, unless indicated otherwise in the credit line; if the material is not included under the Creative Commons license, users will need to obtain permission from the license holder to reproduce the material. To view a copy of this license, visit http://creativecommons.org/licenses/ by/4.0/

(c) The Author(s) 2016

Supplementary Information accompanies the paper on the npj Biofilms and Microbiomes website (http://www.nature.com/npjbiofilms) 